\title{
Impactos ambientais de lixão a céu aberto no Município de Cristalândia, Estado do Piauí, Nordeste do Brasil
}

\section{Tancio Gutier Ailan Costa ${ }^{1 *}$, Bruna de Freitas Iwata ${ }^{2}$, Camila Portela de Castro ${ }^{1}$, Juliana Vogado Coelho ${ }^{1}$, Gleide Ellen dos Santos Clementino $^{1}$, Laécio Miranda Cunha ${ }^{1}$}

${ }^{1}$ Graduando do Curso de Tecnologia em Gestão Ambiental. Instituto Federal de Educação, Ciência e Tecnologia do Piauí. Campus Corrente. Rua 6, S/No. Nova Corrente. Corrente-PI (CEP 64980-000).*E-mail: gutierailan@gmail.com.

2Docente do Curso de Tecnologia em Gestão Ambiental. Instituto Federal de Educação, Ciência e Tecnologia do Piauí. Campus Corrente. Rua 6, S/Nº Nova Corrente. Corrente-PI (CEP 64980-000). E-mail: iwata@ifpi.edu.br.

Resumo. O processo de criação e ampliação das áreas urbanas ao longo dos anos tem contribuído para o aparecimento de diversos impactos ambientais negativos ao meio ambiente. $\mathrm{O}$ surgimento e o desenvolvimento da sociedade sempre estiveram relacionados à geração de resíduos sólidos oriundos de diversas atividades, sendo estes considerados um dos principais causadores de impactos ambientais. Desta forma, considerando as problemáticas ambientais causadas pela má destinação e disposição final inadequada dos resíduos sólidos, o presente estudo teve por objetivo avaliar os impactos ambientais do lixão a céu aberto localizado no Município de Cristalândia-PI (Nordeste do Brasil), mediante o método matricial de avaliação de impactos ambientais. O estudo foi realizado através de pesquisa de campo, com observações diretas sob a área de estudo, visando a diagnosticar a situação da área diretamente afetada. Quanto ao método de avaliação de impactos ambientais utilizou-se como base a Matriz de Leopold, para identificar e descrever os possíveis impactos ambientais ocorridos, assim como o levantamento fotográfico. Observou-se que dentre os principais impactos ambientais, causados na área estão o aumento do processo erosivo, a compactação do solo, a emissão de gases de efeito estufa, a contaminação do solo e do lençol freático, a redução e o estresse da fauna local, a redução da biodiversidade nativa, entre outros. No caso analisado, fica evidenciado que o lixão do Município de Cristalândia possui um caráter degradativo em relação aos recursos naturais locais, prejudicando assim a qualidade ambiental da área diretamente afetada. (IFPI).

Palavras-chave: Resíduos sólidos; Lixão; Destinação final; Planejamento urbano.

Abstract. Environmental impacts of dumpsite in open sky in the Municipality of Cristalândia-PI, Northeast Brazil. The process of creation and expansion of urban areas over the years has contributed to the emergence of several negative environmental impacts to the
Recebido:

30/05/2015

Aceito:

27/06/2016

Publicado:

30/06/2016

Acesso Aberto Artigo completo

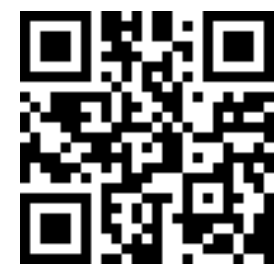

ORCID

(ㄱ) 0000-0002-0635-2651

Tancio Gutier Ailan

Costa

D) 0000-0002-6465-9731

Bruna de Freitas Iwata

(1) 0000-0002-9928-1863

Camila Portela de

Castro

이 0000-0001-5264-8499

Gleide Ellen dos Santos Clementino

0000-0003-2029-9997 Juliana Vogado Coelho

(1) 0000-0002-8147-8019

Laécio Miranda Cunha 
environment. The emergence and development of society have always been related to the generation of solid waste from various activities, which are considered a major cause of environmental impacts. In this way, considering the environmental problems caused by solid waste, this study aimed to evaluate the environmental impacts on landfill will open in the Municipality of Cristalândia, State of Piauí, Northeast Brazil) through the matrix method of environmental impact assessment. The study was conducted through field research with direct observations in the area in order to diagnose the situation directly affected the area. As for the method of assessment of environmental impacts was used based on the Leopold Matrix, to identify and describe the potential environmental impacts that have occurred, as well as photographic survey. It was noted that among the main environmental impacts caused in the area are increased erosion, soil compaction, emission of greenhouse gases, groundwater and soil contamination, reducing stress and the local wildlife, reduced native biodiversity, among others. In this case, it is evident that the landfill located in Municipality of Cristalândia has a degradative character in relation to local natural resources, thus undermining the environmental quality of the area directly affected. (IFPI)

Keywords: Solid waste; Garbage dump; Final destination; urban planning.

\section{Introdução}

O processo de criação e ampliação das áreas urbanas ao longo dos anos tem contribuído para o aparecimento de diversos impactos ambientais negativos ao meio ambiente. O surgimento e o desenvolvimento da sociedade sempre estiveram relacionados à geração de resíduos sólidos oriundos de diversas atividades, sendo considerados um dos principais causadores de impactos ambientais atualmente.

Mesmo sendo um problema mundial, as consequências negativas da alta produção de resíduos sólidos são mais perceptíveis nos países em desenvolvimento. Os países mais ricos são os maiores produtores, entretanto, possui uma maior capacidade de equacionamento da gestão, por um somatório de fatores que incluem recursos econômicos, preocupação ambiental da população e desenvolvimento tecnológico. Em cidades de países em desenvolvimento com urbanização muito acelerada, verificam-se déficits na capacidade financeira e administrativa dessas em prover infraestrutura e serviços essenciais como água, saneamento, coleta e destinação adequada do lixo e moradia, e em assegurar segurança e controle da qualidade ambiental para a população (Jacobi e Besen, 2011).

Especificamente no Brasil, o manuseio e eliminação de resíduos sólidos é um dos fatores de maior contribuição na geração de impactos ambientais, que coloca em risco principalmente a saúde pública. A falta de locais adequados para disposição final dos RS ainda é uma problema enfrentado pela maioria dos municípios brasileiros. Segundo dados obtidos na pesquisa nacional de saneamento básico, realizada pelo Instituto Brasileiro de Geografia e Estatística, cerca de 50,8\% dos resíduos produzidos no país ainda são lançados em vazadouros a céu aberto. $\mathrm{O}$ problema é mais agravante na região norte do país, onde aproximadamente 59\% dos municípios lançam seus resíduos em lixões (Abrelpe, 2010).

A disposição final dos resíduos sólidos urbanos dentro do município é de responsabilidade das prefeituras, exceto os resíduos de caráter especial que deverá ser de responsabilidade do próprio gerador. No entanto, a grande maioria dos resíduos 
sólidos gerados no Brasil é disposta em lixões causando sérios problemas ambientais.

Neste contexto, quando dispostos de forma inadequada, os resíduos sólidos podem causar poluição da água, do ar e do solo, além de criar ambiente propício para a proliferação de macro e micro vetores causadores de doenças (Besen, 2011), com consequências desastrosas, tanto para 0 meio ambiente quanto para a qualidade de vida da população.

Silva et al. (2012) elenca o aterro controlado como uma das técnicas de minimização de impactos ambientais, causados por lixões a céu aberto, uma vez que são utilizados alguns métodos de engenharia para confinar os resíduos sólidos, cobrindo com uma camada de material inerte na conclusão de cada jornada de trabalho. Entretanto, ainda sim, produz poluição, principalmente quando não dispõe de impermeabilização do solo, que pode comprometer a qualidade das águas subterrâneas e de sistemas de tratamento de percolados e de biogás gerado (Brasil, 2006).

Partindo da insegurança da utilização de técnicas de disposição final dos resíduos sólidos, surge à necessidade de avaliar os reais impactos causados pela prática inadequada de descarga de resíduos a céu aberto. Uma vez que a avaliação de impacto ambiental evidencia os efeitos ecológicos, econômicos e sociais que advêm da implantação de atividades antrópicas, bem como de monitoramento e controle desses efeitos pelo poder público e pela sociedade (Silva et al., 2012).

Desta forma, considerando as problemáticas ambientais causadas pelos resíduos sólidos, o presente estudo teve por objetivo avaliar os impactos ambientais de lixão a céu aberto localizado no Município de Cristalândia Piauí, mediante o método matricial de avaliação de impactos ambientais.

\section{Metodologia}

\section{Área de estudo}

$\mathrm{O}$ estudo foi realizado no Município de Cristalândia, localizada no extremo sul do Estado do Piauí $\left(10^{\circ} 39^{\prime} 11^{\prime \prime}\right.$ S e $\left.45^{\circ} 11^{\prime} 06^{\prime \prime} \mathrm{W}\right)$, estando a uma altitude de $469 \mathrm{~m}$ (IBGE, 2010). O município compreende uma área de aproximadamente $1.203 \quad \mathrm{~km}^{2}$, com população estimada em 7.831 habitantes. $\mathrm{O}$ clima da região é o tropical úmido, de acordo a classificação de Köppen, com período chuvoso concentrado principalmente entre os meses de fevereiro e abril, localizando-se no Bioma Cerrado (Figura 1). A pesquisa foi desenvolvida no lixão a céu aberto distando aproximadamente $4 \mathrm{~km}$ da sede do município.

\section{Procedimentos metodológicos}

O estudo foi realizado através de pesquisa de campo, com observações diretas sob área de estudo, visando a diagnosticar a situação atual do lixão a céu aberto. Para tanto, utilizou-se de levantamento fotográfico, buscando representar os possíveis impactos ambientais ocasionados pelo lixão. Quanto ao método de avaliação de impactos ambientais, utilizou-se como base a Matriz de Leopold et al. (1971), para identificar e descrever os possíveis impactos ambientais ocorridos na área. Foram realizadas adaptações particulares aos impactos ambientais do lixão, seguindo a matriz qualitativa de impactos ambientais, de acordo com Santos (2004) e Sobral et al. (2007). Os parâmetros qualitativos foram avaliados de acordo as seguintes características:

Frequência: remete ao padrão de ocorrência do impacto, que pode ser caracterizado como temporário (T), quando o efeito do impacto manifesta-se por um determinado tempo após a realização da ação; permanente (Pr), quando, uma vez 
executada a ação, os efeitos continuam a manifestar-se num horizonte temporal conhecido; ou cíclico (C), quando o efeito é sentido em determinados períodos (ciclos), que podem ser ou não constatado ao longo do tempo.

Extensão: é o alcance do impacto, que pode ser caracterizado como: local (L), quando o efeito circunscreve-se ao próprio local da ação; ou regional (Rg), quando o efeito se propaga por uma área além das imediações da localidade onde se dá a ação.

Duração: refere-se ao tempo que o impacto e seus efeitos persistem no ambiente, podendo ser caracterizado com de curto prazo (Cp), quando seus efeitos têm duração de até um ano; de médio prazo (Mp), quando seus efeitos têm duração de um a 10 anos; e de longo prazo (Lp), quando seus efeitos têm duração de 10 a 50 anos.

Sentido: refere-se à valoração do impacto, ou seja, se o impacto é caracterizado como: positivo (P), quando a ação impactante causa melhoria da qualidade de um parâmetro ambiental; ou negativo $(\mathrm{N})$, quando uma ação causa um dano à qualidade ambiental.

Grau de impacto: é o critério de classificação usado para indicar a gravidade do impacto no meio ambiente, que pode ser baixo (B), quando a utilização dos recursos naturais é desprezível quanto ao seu esgotamento e à degradação do meio ambiente e da comunidade, sendo desprezível e reversível; médio (M), quando a utilização de recursos naturais é considerada sem que haja possibilidade de esgotamento das reservas naturais, sendo a degradação do meio ambiente e da comunidade reversível, porém com ações imediatas; alto (A) quando a ação provoca a escassez de recursos naturais, a degradação do meio ambiente e da comunidade, não tendo muitas probabilidades de reversibilidade.

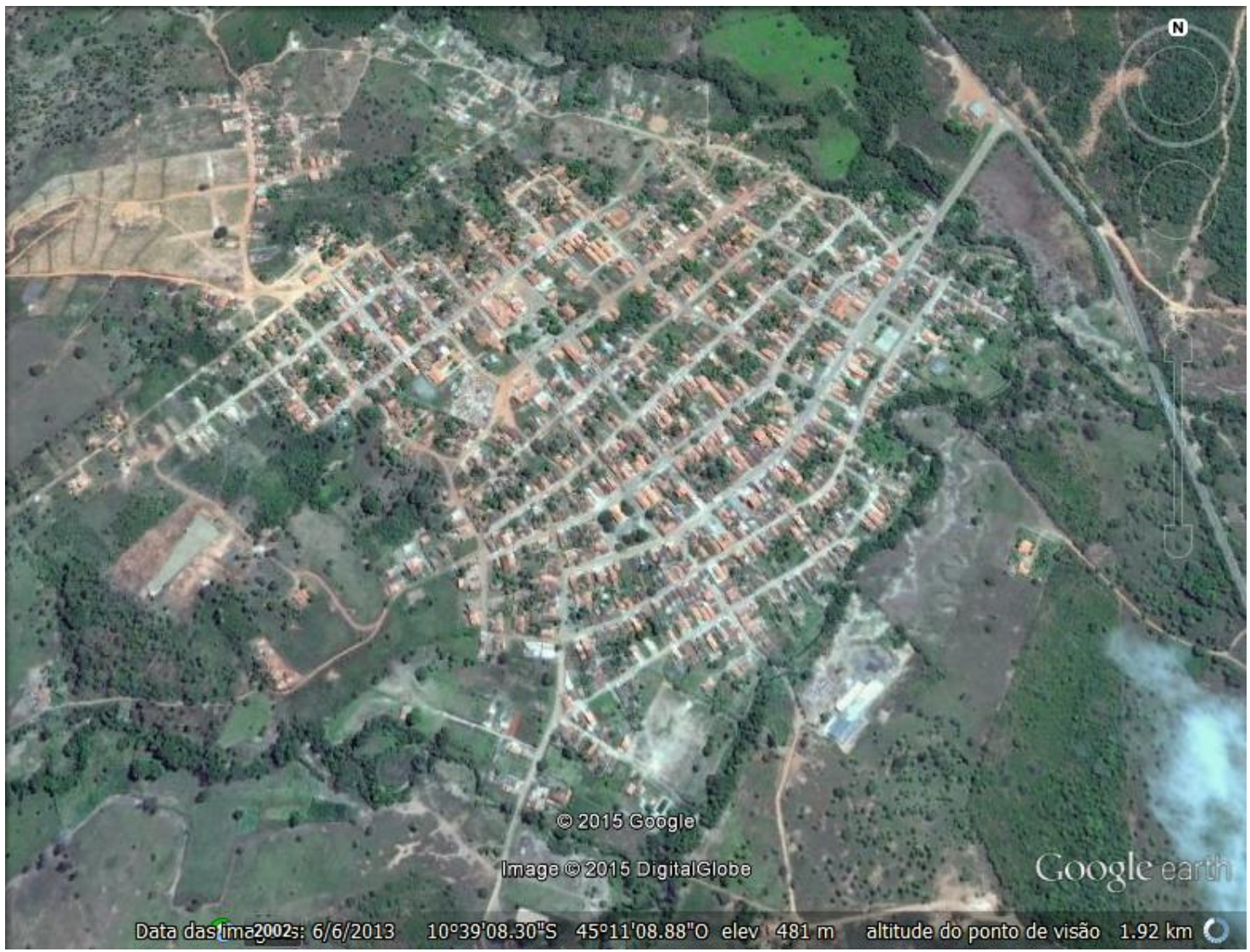

Figura 1. Imagem de satélite do Município de Cristalândia-PI, 2013. Fonte: Google Earth, 2015. 


\section{Resultados e discussão}

Durante toda a pesquisa de campo, pôde-se observar que a maior parte dos resíduos sólidos dispostos no lixão era constituída basicamente de papeis, papelão, madeira, plástico e resíduos orgânicos. Os resíduos gerados no hospital do município são coletados pela prefeitura e conduzidos para o lixão. Esse tipo de resíduo, apesar de não ser a melhor medida a ser tomada, deveria ser separado e aterrado imediatamente, em local reservado.

A forma de disposição de resíduos sólidos a céu aberto favorece a proliferação de micro e macrovetores, os quais podem ser via de acesso de organismos patogênicos, que pode causar problemas à saúde dos indivíduos que se apresentem no local. Entretanto, não foi identificada a presença de catadores no lixão (Figura 2).

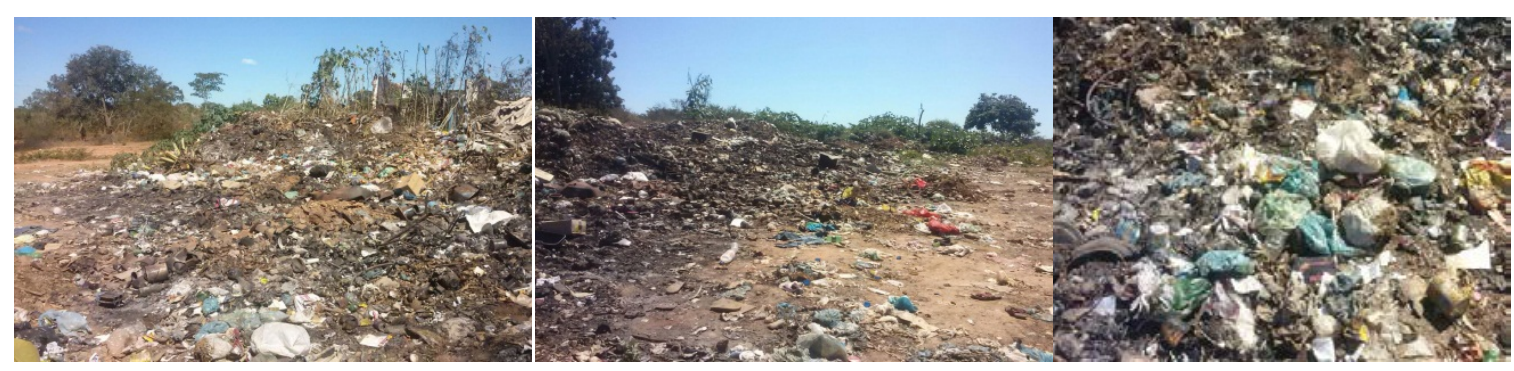

Figura 2. Disposição de resíduos sólidos no lixão a céu aberto no Município de Cristalândia, Piauí.

Outro problema identificado foi a poluição visual das áreas circunvizinhas, produzindo alterações da paisagem por longas extensões. Segundo Leite e Lopes (2000) em um lixão existem também a poluição visual das áreas circunvizinhas pelos resíduos leves, como plásticos e papeis que são conduzidos pelo vento por uma longa distância.

Além desses, os principais impactos identificados no lixão analisados foram, aumento do processo erosivo, compactação do solo, emissão de gases de efeitos estufa, contaminação do solo e possivelmente do lençol freático, redução e estresse da fauna local, redução da biodiversidade nativa. Esses impactos afetam a qualidade ambiental e promovem um desequilíbrio ecológico para os animais vivos que habitam nessa área, especificamente no que diz respeito à fauna e flora local, como identificado na pesquisa. A Tabela 1 contém os principais impactos identificados.

O estudo verificou através da análise matricial dos impactos ambientais que o lixão do município possui um caráter degradativo, uma vez que seus impactos afetam o ambiente de forma negativa, comprometendo o equilíbrio do ecossistema no qual o lixão está inserido. Esta ação negativa pode ser observada claramente pela a análise dos aspectos descritos na Tabela 1, na qual a maioria dos impactos apresenta alta capacidade de degradação.

Os efeitos desses impactos ocorrem de forma permanente, pois uma vez executada a ação os efeitos continuam a manifestar-se por escala temporal indeterminada. Isso pode ser evidenciado pela frequente ocorrência de aumento dos processos erosivos, que foram originados principalmente pela remoção da vegetal para abertura do local. Segundo Sánchez (2008) o avanço dos processos erosivos é determinado pela integração de vários fatores, destacando o tipo de solo, a topografia do terreno, o clima local, as formas de ocupação do território e também pelo tipo de cobertura vegetal. 
Tabela 1. Matriz de avaliação qualitativa dos impactos ambientais do lixão a céu aberto do Município de Cristalândia, Piauí.

\begin{tabular}{|c|c|c|c|c|c|c|c|c|c|c|c|c|c|}
\hline \multirow{3}{*}{ Impactos Ambientais } & \multicolumn{13}{|c|}{ Características } \\
\hline & \multicolumn{3}{|c|}{ Frequência } & \multicolumn{2}{|c|}{ Extensão } & \multicolumn{3}{|c|}{ Duração } & \multicolumn{2}{|c|}{ Sentido } & \multicolumn{3}{|c|}{$\begin{array}{l}\text { Grau de } \\
\text { Impacto }\end{array}$} \\
\hline & $\mathbf{T}$ & Pr & $\mathbf{C}$ & $\mathbf{L}$ & Rg & Cp & Mp & $\mathbf{L p}$ & $\mathbf{P}$ & $\mathbf{N}$ & $\mathbf{B}$ & $\mathbf{M}$ & $\mathbf{A}$ \\
\hline & \multicolumn{13}{|c|}{ Meio Físico } \\
\hline Aumento dos processos erosivos & & $\mathrm{x}$ & & $\mathrm{x}$ & & & & $\mathrm{x}$ & & $\mathrm{x}$ & & & $\mathrm{x}$ \\
\hline Compactação do solo & & $\mathrm{X}$ & & $\mathrm{x}$ & & & $\mathrm{x}$ & & & $\mathrm{X}$ & & & $\mathrm{x}$ \\
\hline Emissão de gases de efeito estufa & & $\mathrm{x}$ & & & $\mathrm{x}$ & & $\mathrm{x}$ & & & $\mathrm{x}$ & & & $\mathrm{x}$ \\
\hline Possível contaminação do solo & & & $\mathrm{x}$ & $\mathrm{x}$ & & & & $\mathrm{x}$ & & $\mathrm{X}$ & & & $\mathrm{x}$ \\
\hline $\begin{array}{l}\text { Possível depreciação do lençol } \\
\text { freático }\end{array}$ & $\mathrm{x}$ & & & $\mathrm{x}$ & & $\mathrm{x}$ & & & & $\mathrm{x}$ & $\mathrm{x}$ & & \\
\hline & \multicolumn{13}{|c|}{ Meio Biótico } \\
\hline Stress da fauna local & $\mathrm{X}$ & & & $\mathrm{x}$ & & $\mathrm{x}$ & & & & $\mathrm{X}$ & $\mathrm{x}$ & & \\
\hline Redução da biodiversidade nativa & & $\mathrm{x}$ & & $\mathrm{x}$ & & & & $\mathrm{x}$ & & $\mathrm{x}$ & & & $\mathrm{x}$ \\
\hline $\begin{array}{l}\text { Redução da capacidade de } \\
\text { sustentação da flora }\end{array}$ & & & $\mathrm{x}$ & $\mathrm{X}$ & & & $\mathrm{x}$ & & & $\mathrm{x}$ & & $\mathrm{x}$ & \\
\hline Redução da biota do solo & & $\mathrm{X}$ & & $\mathrm{x}$ & & & & $\mathrm{x}$ & & $\mathrm{X}$ & & & $\mathrm{x}$ \\
\hline & \multicolumn{13}{|c|}{ Meio Antrópico } \\
\hline Poluição visual & & $\mathrm{X}$ & & $\mathrm{x}$ & $\mathrm{x}$ & & & $\mathrm{x}$ & & $\mathrm{x}$ & & $\mathrm{x}$ & \\
\hline Proliferação de doenças & & $\mathrm{X}$ & & $\mathrm{x}$ & $\mathrm{x}$ & & $\mathrm{x}$ & $\mathrm{x}$ & & $\mathrm{x}$ & & & $\mathrm{X}$ \\
\hline
\end{tabular}

*T - Temporário; Pr - Permanente; C - Cíclico; L - Local; Rg - Regional; Cp - Curto prazo; Mp - Médio prazo; Lp - Longo prazo; P - Positivo; N - Negativo; B - Baixo; M - Médio; A - Alto.

A grande maioria dos resíduos dispostos no lixão é submetida à queima, sendo uma estratégia utilizada para reduzir a quantidade de resíduos acumulados no local (Figura 3). Esse processo de queima acaba agredindo ainda mais o meio ambiente, uma vez que a combustão desses resíduos emite grandes quantidades de

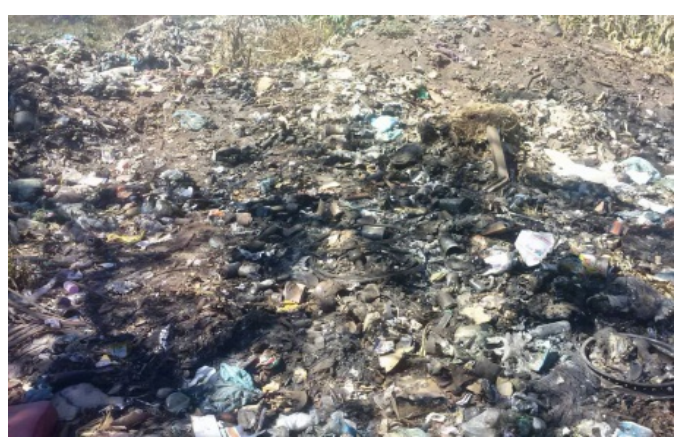

gases de efeito estufa, contribuindo com a poluição do ar do município. Além da própria deposição dos resíduos no lixão, o processo de queima também contribui para a redução da biodiversidade, uma vez que o fogo afasta os animais e afeta os vegetais ali presentes.

Figura 3. Representação da queima de resíduos sólidos no lixão a céu aberto no município.

Do ponto de vista da extensão, a maioria dos impactos foram considerados local, não se estendendo por grandes áreas tampouco atingindo indiretamente as cidades vizinhas ao município. Por exemplo, a proliferação dos vetores como baratas, moscas e ratos, poluição do solo e possivelmente do lençol freático (Figura 3). 
Os processos de degradação dos recursos naturais ocorridos no lixão a céu aberto no Município de Cristalândia são provocados pela disposição inadequada dos resíduos sólidos, os quais, conforme a Lei $\mathrm{n}^{\mathrm{o}} 12.305 / 2010$, deveriam ser dispostos em aterros sanitários, sendo a maneira mais correta para a disposição final.

\section{Conclusão}

A disposição dos resíduos sólidos no Município de Cristalândia representa uma ameaça aos recursos ambientais locais, principalmente no que tange à qualidade ambiental do solo, do ar e da água subterrânea. Verifica-se, no município, a presença de um lixão a céu aberto como principal área de destino final dos resíduos urbanos, que por sua vez não apresenta infraestrutura necessária para a proteção dos componentes ambientais (solo, água, ar e sociedade).

É necessário que a gestão municipal tome providências cabíveis para essa problemática da disposição irregular dos resíduos sólidos, conforme o que preconiza a Lei $\mathrm{n}^{\circ}$ 12.305/2010 (Política Nacional de Resíduos Sólidos) (Brasil, 2010), buscando a adoção de medidas que minimizem as possibilidades de degradação ambiental, como a criação de um aterro sanitário no município, como uma forma adequada de disposição final dos resíduos sólidos.

\section{Agradecimentos}

Ao Instituto Federal de Educação, Ciência e Tecnologia do Piauí pelo apoio à execução desse estudo.

\section{Declaração de conflito de interesses}

Os autores declaram não haver conflito de interesses.

\section{Referências}

Abrelpe - Associação Brasileira de Empresas de Limpeza Pública e Resíduos Especiais. Panorama dos Resíduos Sólidos no Brasil. 2010. Disponível em:
<http://www.abrelpe.org.br/downloads/Panoram a2010.pdf>. Acesso em: 19 maio 2015.

Brasil. Fundação Nacional de Saúde. Manual de saneamento. 3. ed. rev. Brasília: Fundação Nacional de Saúde, 2006.

Brasil. Leis, decretos, etc. Lei $\mathbf{n}^{0} \mathbf{1 2 . 3 0 5}$, de 2 de agosto de 2010. Institui a Política Nacional de Resíduos Sólidos; altera a Lei $n^{0}$ 9.605, de 12 de fevereiro de 1998; e dá outras providências. Disponível em: <http://www.planalto.gov.br/ ccivil_03/_ato2007-2010/2010/lei/112305.htm>. Acesso em: 19 maio 2015.

Besen, G. R. Coleta seletiva com inclusão de catadores: construção participativa de indicadores e índices de sustentabilidade. São Paulo: Universidade de São Paulo, 2011. (Tese Doutorado).

IBGE - Instituto Brasileiro de Geografia e Estatística. Censo Demográfico 2010: sinopse. Rio de Janeiro: IBGE, 2010. Disponível em: <http://censo2010.ibge.gov.br/>. Acesso em: 19 maio 2015.

Jacobi, P. R.; Besen, G. R. Gestão de resíduos sólidos em São Paulo: desafios da sustentabilidade. Estudos Avançados, v. 25, p. 135-158, 2011. http://dx.doi.org/10.1590/S010340142011000100010

Leite, V. D.; Lopes, W. S. Avaliação dos aspectos sociais, econômicos e ambientais causados pelo lixão da Cidade de Campina Grande-PB. Anais do IX Simpósio LusoBrasileiro de Engenharia Sanitária e Ambiental, Porto Seguro, 2000, p. 1534-1540.

Leopold, L. B.; Clarke, F. E.; Hanshaw, B. B.; Balsley, J. R. A procedure for evaluating environmental impact. Washington: United States Department of the Interior, Geological Survey, 1971. (Geological Survey Circular, 645). Disponível em: <http://eps.berkeley.edu/ people/lunaleopold/(118) A Procedure for Evaluating Environmental Impact.pdf $>$. Acesso em: 19 maio 2015.

Sanchez, L. E. Avaliação de Impacto Ambiental: conceitos e métodos. São Paulo: Oficina de Textos, 2006.

Sanchez, L. E. Avaliação de Impacto Ambiental: conceitos e métodos. São Paulo: Oficina de Textos, 2008.

Santos, R. F. Planejamento ambiental: teoria e prática. São Paulo: Oficina de Textos, 2004.

Silva, S. A. F.; Aragão, M. H. S.; Silva, G. A. B.; Silva, T. S.; Almeida, M. M.; Souza, N. C. Caracterização de impactos ambientais causados por um vazadouro na Cidade de Mogeiro-PB. Anais do $1^{\circ}$ Encontro Nacional de 
Educação, Ciência e Tecnologia, Campina Grande, 2012. Disponível em: $<$ http://www.editorarealize.com.br/revistas/enec t/trabalhos/Poster_301.pdf $>$. Acesso em: 19 maio 2015.

Sobral, I. S.; Santana, R. K. O.; Gomes, L. J.; Ribeiro, G. T.; Santos, J. R.; Costa, M. Avaliação dos impactos ambientais no Parque Nacional Serra de Itabaiana-SE. Revista Caminhos de Geografia, v. 8, n. 24, p. 102110, 2007. Disponível em: $<$ http://www.seer.ufu.br/index.php/caminhosdeg eografia/article/download/15713/8888>. Acesso em: 19 maio 2015.

Informação da Licença: Este é um artigo Open Access distribuído sob os termos da Licença Creative Commons Attribution, que permite uso irrestrito, distribuição e reprodução em qualquer meio, desde que a obra original seja devidamente citada. 